\title{
Neurologic and histologic outcomes after intrathecal injection of bupivacaine liposome injectable suspension in pigs: A pilot study
}

Zel J ${ }^{1}$, Hadzic A ${ }^{2,3}$, Cvetko E ${ }^{4}$, Seliskar A ${ }^{1}$, Damjanovska $\mathrm{M}^{5}$, Kuroda $\mathrm{MM}^{2}$, Stopar Pintaric $\mathrm{T}^{4,5}$

1: Small Animal Clinic, Veterinary Faculty, University of Ljubljana, Ljubljana, Slovenia; 2: NYSORA (The New York School of Regional Anaesthesia), New York, NY, USA; 3: Department of Anaesthesiology, Ziekenhuis Oost-Limburg, Genk, Belgium; 4: Institute of Anatomy, Faculty of Medicine, University of Ljubljana, Korytkova 2, Ljubljana; 5: Clinical Department of Anaesthesiology and Intensive Therapy, University Medical Centre Ljubljana, Ljubljana, Slovenia

\section{Background}

Extended-release local anesthetic formulations have been developed to prolong the duration of neural blockade. Bupivacaine liposome injectable suspension (EXPAREL ${ }^{\circledR}$ ) is an extended release formulation of bupivacaine encapsulated in multivesicular liposomes, that has been approved by the US Food and Drug Administration for postoperative analgesia via tissue infiltration, and for interscalene brachial plexus nerve blocks. In clinical studies using liposome bupivacaine in nerve blocks, infiltration of liposome bupivacaine into the surgical site of several soft tissue and orthopedic surgery models provided analgesia with reduced opioid consumption for up to $72 \mathrm{~h}$. Extended sensory block without apparent neurologic complications has been reported after epidural injection of liposome bupivacaine in humans. However, there are limited data on the intrathecal application of liposome bupivacaine. The paucity of protective connective tissue in the intrathecal space renders the delicate neural structures vulnerable to direct prolonged exposure to injected anesthetics and to increased risk for toxicity.

This pilot study describes the neurologic and histologic outcomes of escalating dosages of liposome bupivacaine suspension administered intrathecally. The study was conducted in a porcine model as the anatomy of the spine in a pig weighing approximately $40 \mathrm{~kg}$ is similar to that of an adult human.

Methods

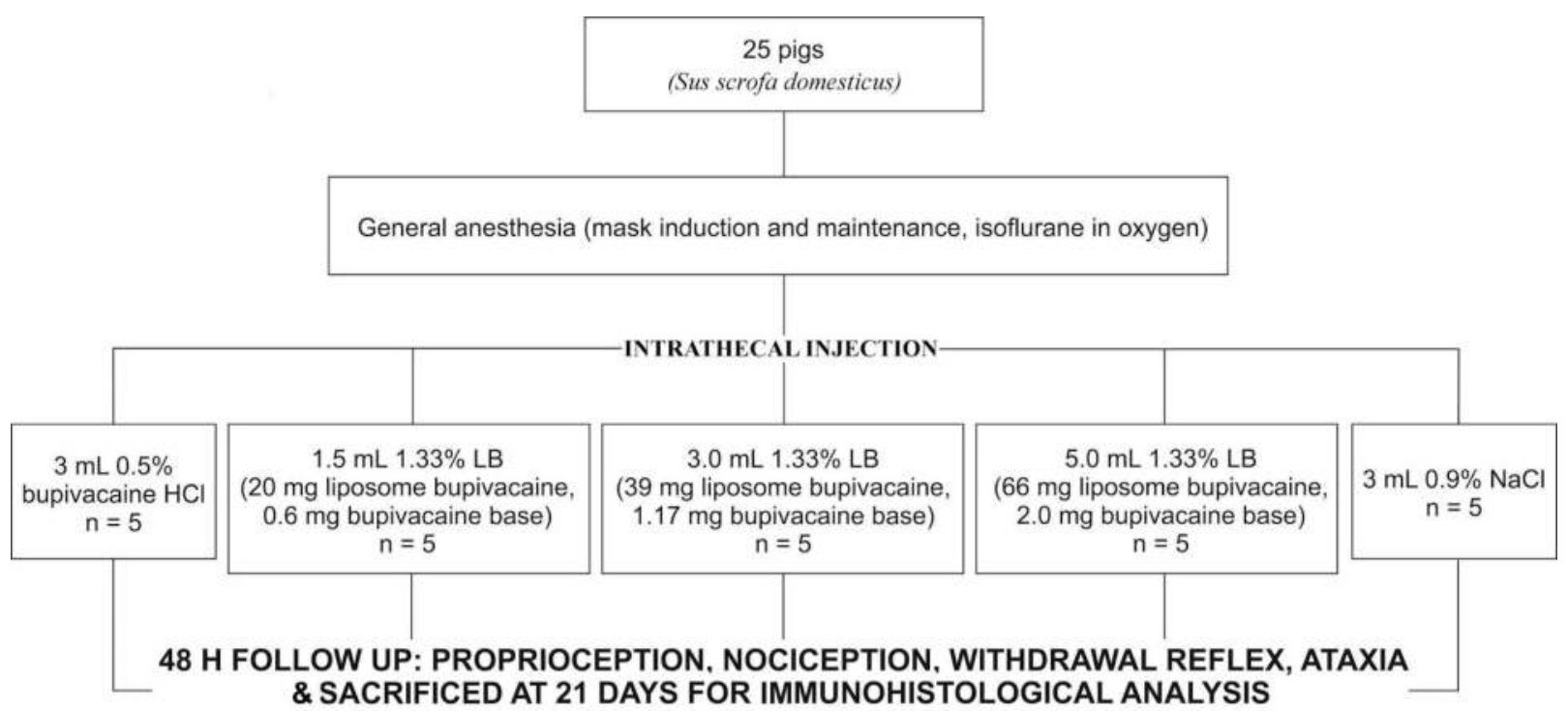

Figure 1:The pigs were randomly assigned to five groups to receive the study drugs intrathecally. The pigs were neurologically examined hourly for the next $12 \mathrm{~h}$, followed by examinations every $4 \mathrm{~h}$ for $48 \mathrm{~h}$. At 21 days the pigs were anesthetized and sacrificed. Samples were taken for detailed immunohistologic analysis.

\section{Results}

Animals that received $0.9 \%$ sodium chloride and bupivacaine $15 \mathrm{mg}$ recovered within 2 hours and 5 hours, respectively. Animals that received the liposome bupivacaine $39 \mathrm{mg}$ or $66 \mathrm{mg}$ doses exhibited signs of neuraxial block (decreased nociception and proprioception) up to 32 hours after injection.

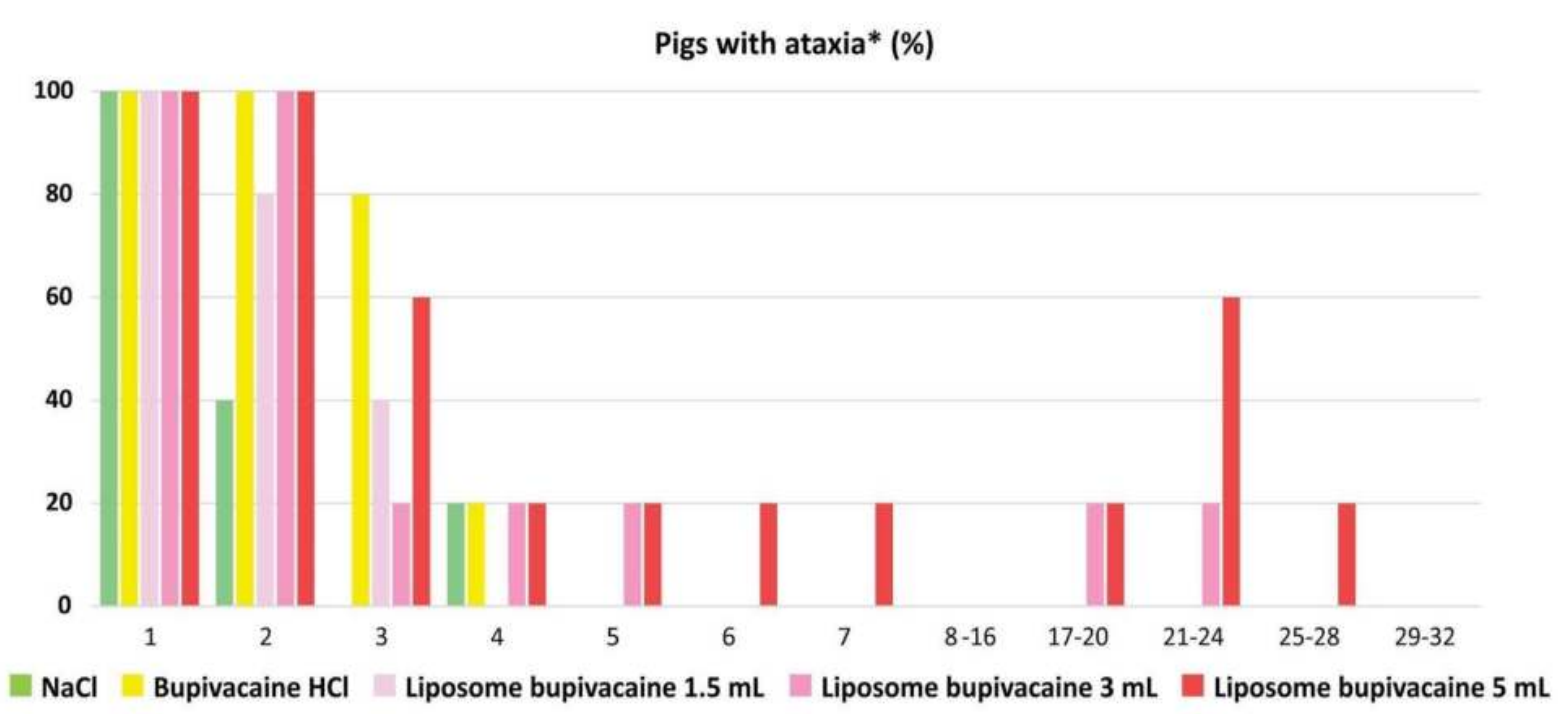

Picture 1: Percentage of pigs showing any grade of ataxia by hours after intrathecal application. * ataxia defined as heavy, slight ataxia or non-ambulatory.

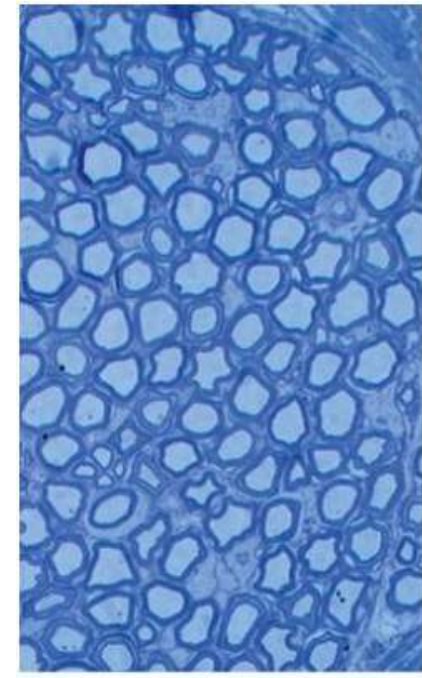

$\mathrm{NaCl} 0.9 \%$

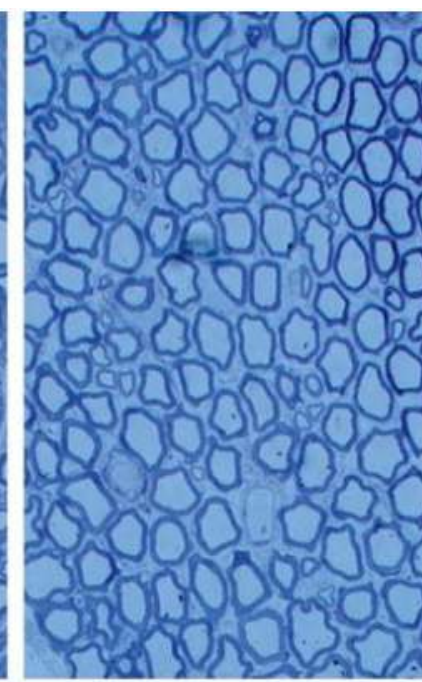

Bupivacaine $\mathrm{HCl}$

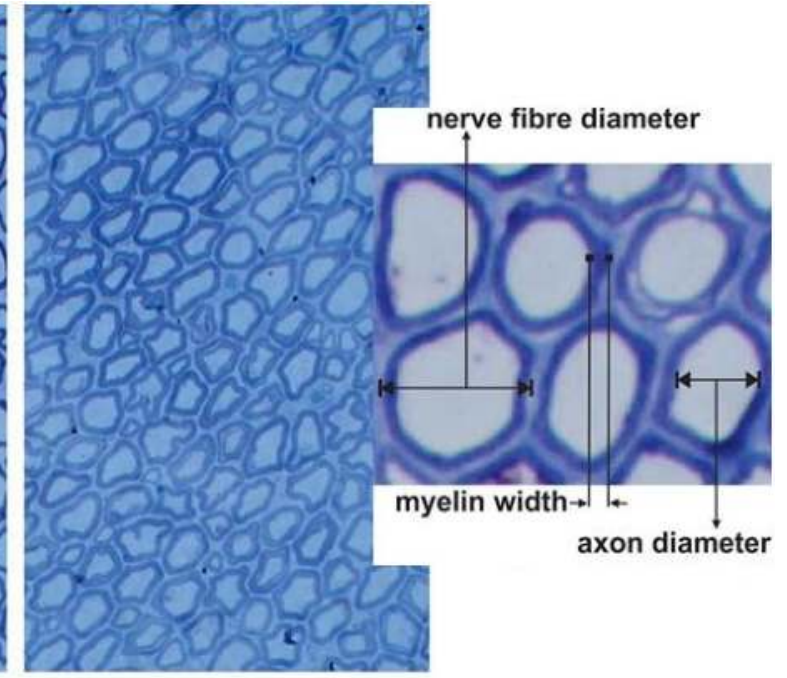

$66 \mathrm{mg}$ liposome bupivacaine

Picture 2: Examples of morphometric analysis cross sections stained with toluidine blue.

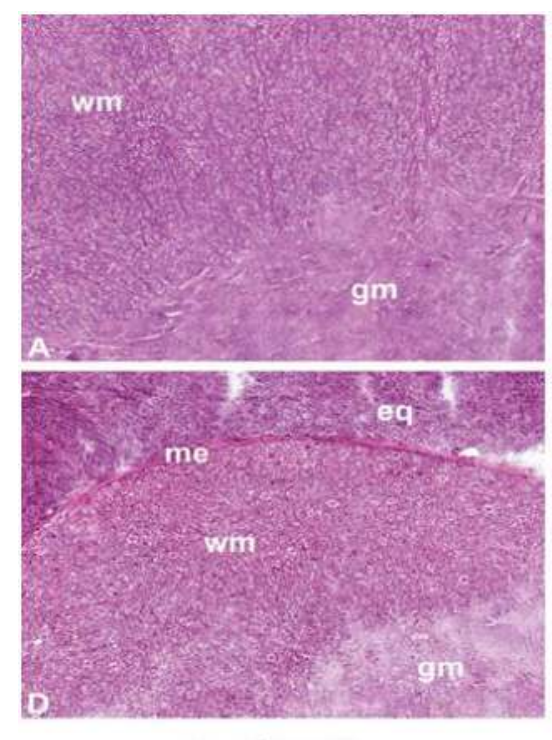

$\mathrm{NaCl} 0.9 \%$

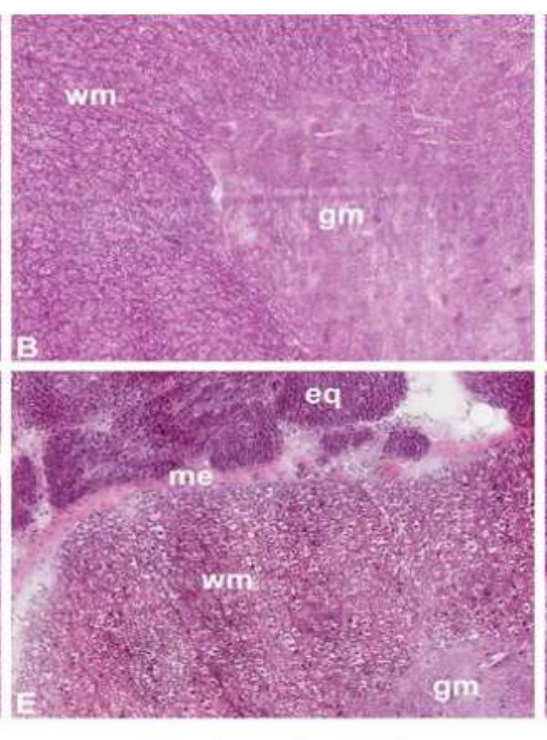

Bupivacaine $\mathrm{HCl}$

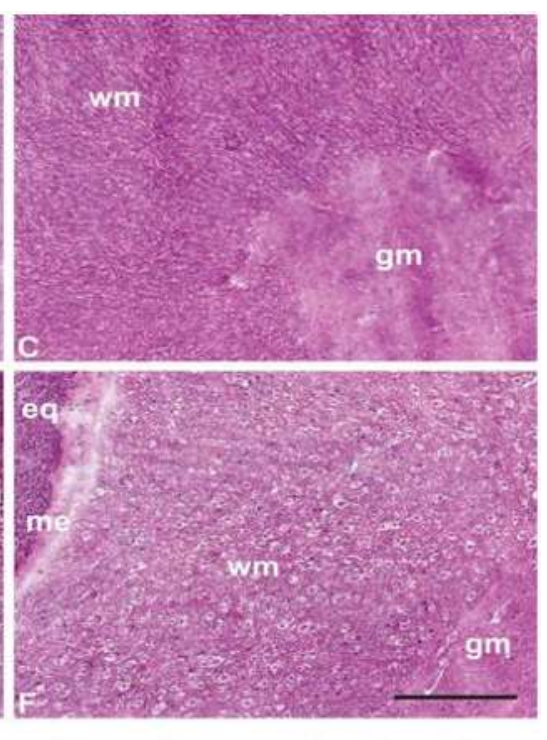

$66 \mathrm{mg}$ liposome bupivacaine
Picture 3: Transverse sections of thoracic (A-C) and lower lumbar (DE) spinal cord show no pathologic changes in gray matter (gm), white matter (wm) or meninges (me) or cauda equina (eq) after injection of saline, bupivacaine $\mathrm{HCl}$ or liposome bupivacaine. Haematoxilin eosin staining, bar $100 \mu \mathrm{m}$.

\section{Conclusions}

Intrathecal administration of liposome bupivacaine injectable suspension in pigs results in a dose-response pattern of longer duration of neuraxial block than bupivacaine hydrochloride without histologic evidence of neurotoxicity. All animals exhibited impairment of neurologic function during the first $2 \mathrm{~h}$ after emergence from general anesthesia, suggesting that the neurologic effects observed were likely due to the drugs used for premedication and general anesthesia. None of the pigs in our study exhibited impairment of the front legs or respiratory depression, suggesting that the dosages and volumes selected for this study are not likely to cause high spinal block in humans, given the anatomical similarities of the pig and human spines. After an initial neural blockade, the decrease in nociception and proprioception in animals that received $66 \mathrm{mg}$ of liposome bupivacaine lasted longer than that in animals that received $3 \mathrm{~mL} 0.5 \%$ preservative-free bupivacaine $\mathrm{HCl}$. Moreover, neural block, returned after $17-20 \mathrm{~h}$ and lasted up to $32 \mathrm{~h}$, suggesting an extended neural block.

The observed neuraxial blockade is consistent with previously published pharmacokinetic data, which suggest that the release of bupivacaine base increases at $12 \mathrm{~h}$ and continues throughout $72+\mathrm{h}$ with maximum concentration occurring between 24-48 h.

However, different degrees impairment in nociception could exist in other dermatomal segments such as the thoracic and abdominal levels. Moreover, slight changes in nociception, which may have clinical relevance, may have been missed by the methods used. Although we did not detect neurologic injury, it is possible that additional methods to assess mitochondrial dysfunction, neuronal apoptosis, neuronal membrane disruption, and cell necrosis could be more sensitive and are suggested for future studies. 